\title{
Point-Spread-Function-Aware Slice-to-Volume Registration: Application to Upper Abdominal MRI Super-Resolution
}

\author{
Michael Ebner ${ }^{1}$, Manil Chouhan ${ }^{2,3}$, Premal A. Patel ${ }^{1,4}$, David Atkinson ${ }^{2}$, \\ Zahir Amin ${ }^{3}$, Samantha Read ${ }^{3}$, Shonit Punwani ${ }^{2,3}$, Stuart Taylor ${ }^{2,3}$, \\ Tom Vercauteren ${ }^{1}$, and Sebastien Ourselin ${ }^{1}$ \\ 1 Translational Imaging Group, CMIC, University College London, London, UK \\ michael.ebner.14@ucl.ac.uk \\ http://cmictig.cs.ucl.ac.uk \\ 2 Centre for Medical Imaging, University College London, London, UK \\ 3 Radiology Department, University College London Hospitals NHS Foundation \\ Trust, London, UK \\ 4 Radiology Department, Great Ormond Street Hospital for Children NHS \\ Foundation Trust, London, UK
}

\begin{abstract}
MR image acquisition of moving organs remains challenging despite the advances in ultra-fast 2D MRI sequences. Post-acquisition techniques have been proposed to increase spatial resolution a posteriori by combining acquired orthogonal stacks into a single, high-resolution (HR) volume. Current super-resolution techniques classically rely on a two-step procedure. The volumetric reconstruction step leverages a physical slice acquisition model. However, the motion correction step typically neglects the point spread function (PSF) information. In this paper, we propose a PSF-aware slice-to-volume registration approach and, for the first time, demonstrate the potential benefit of Super-Resolution for upper abdominal imaging. Our novel reconstruction pipeline takes advantage of different MR acquisitions clinically used in routine MR cholangiopancreatography studies to guide the registration. On evaluation of clinically relevant image information, our approach outperforms state-of-theart reconstruction toolkits in terms of visual clarity and preservation of raw data information. Overall, we achieve promising results towards replacing currently required $\mathrm{CT}$ scans.
\end{abstract}

Keywords: Super-Resolution Reconstruction, Point Spread Function, Registration, Scattered Data Approximation, MRCP Study

\section{Introduction}

In recent years, volumetric magnetic resonance (MR) reconstruction and analysis of moving body organs have attracted increasing clinical interest in numerous areas where subject motion cannot be avoided but the excellent tissue contrast of MR imaging (MRI) is still required. In this context, ultra-fast 2D MRI is 
the method of choice for many applications $[7,13,15]$. However, a balance has to be struck between a short scanning time to avoid motion artefacts, and the signal-to-noise ratio which must be maintained at an acceptable level.

MR cholangio-pancreatography (MRCP) is one typical use of ultra-fast 2D $\mathrm{MR}$ and provides a series of sequences to define the upper gastrointestinal tract and particularly the biliary anatomy [1]. Typically, one axial and one coronal single-shot T2-weighted stack of low-resolution (LR) slices are acquired at inhaled breath-hold. Even though this provides valuable anatomical information, the anisotropic voxel dimensions with their inherently large slice thickness come at a cost. Small structures relevant for clinical diagnosis can be obscured due to partial volume averaging effects (PVEs). Inter-slice motion during image acquisition also limits geometric integrity of the corresponding stack of bundled slices. Additionally, a heavily T2-weighted volume, gated by respiratory motion, is acquired at high resolution. The gain in resolution of liquid-filled structures comes at the cost of structural information from the surrounding structures compared to single-shot slice, as shown in fig. 1 . Consequently, MR alone may not be sufficient for diagnosis and additional contrast-enhanced computed tomography (CT) imaging at higher resolution is performed. However, CT does not have the inherent high soft tissue contrast resolution available on T2-weighted MRI and carries risks of radiation exposure, iodinated contrast exposure and the need for additional investigation increases healthcare costs.

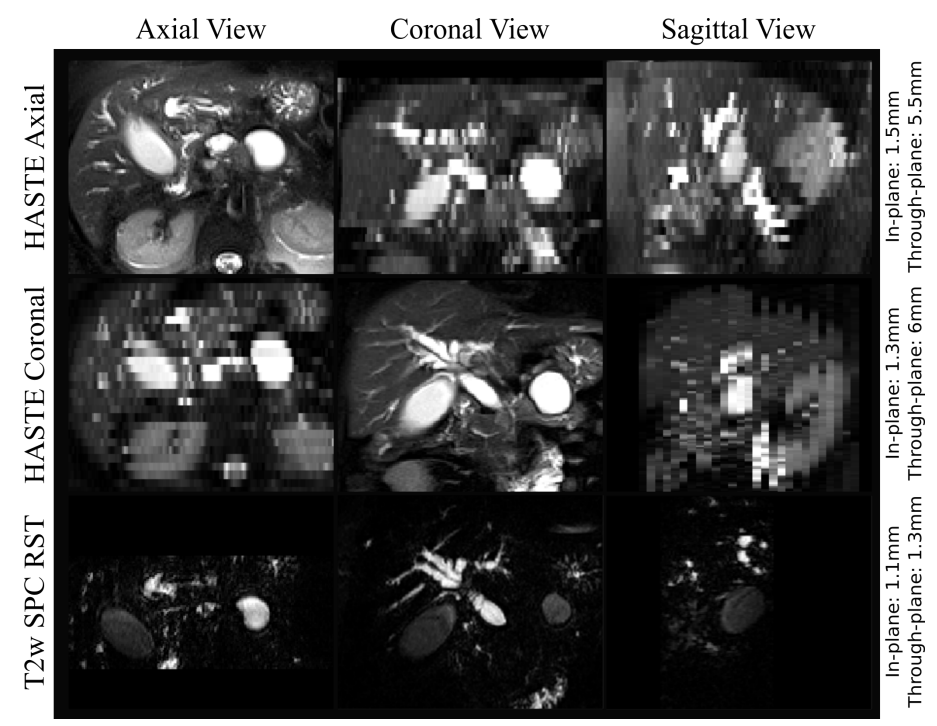

Fig. 1. Visualization of typical MR data acquired in MRCP studies showing anatomy of the biliary tree. Motion is visible throughout the HASTE stacks. The heavily T2weighted volume (T2w SPC RST) has approximately five times higher resolution compared to the HASTE through-plane direction. However, the heavily T2-weighted volume loses valuable tissue contrast in the surrounding anatomy. 
Recent advances in image post-processing have demonstrated the potential to increase the resolution a posteriori by combining several orthogonal MRI stacks of LR 2D slices into a single, HR 3D volume - a method called SuperResolution Reconstruction (SRR). Its application ranges from adult studies on the tongue [15] and thorax [13] to fetal applications [7,10]. To our knowledge, Super-Resolution (SR) has not yet been applied to MRCP studies to define upper gastrointestinal tract and biliary anatomy. An SRR technique needs to overcome several challenges in this context. Firstly, stacks are acquired consecutively and cannot be regarded as motion-free given the non-periodic respiratory motion [8], tissue deformation due to cardiac motion and arterial pulsation, peristaltic and other complex motion affecting the upper gastrointestinal anatomy, as shown in fig. 1. Secondly, accurate registration and reconstruction are complicated by the fact that in current clinical protocols usually only two single-shot T2-weighed stacks are available (in axial and coronal planes) with a slice thickness approximately five times higher than the in-plane resolution. Existing respiratory motion models require the availability of respiratory surrogate data [8] which are currently not available for MRCP studies. Using an SRR approach such as the iterative two-step registration-reconstruction approach used in fetal MRI [7,10], applied to only two stacks, is prone to generate a strongly biased volume and the currently used rigid motion models might not be sufficient. Additionally, current motion correction techniques do not take into account the PSF for registration. This is particularly problematic since neglecting the PSF during resampling introduces aliasing and subsequently results in additional loss of information $[2$, $3]$.

In this paper, our contributions are three-fold: i) introduction of a novel PSFaware slice-to-volume registration (SVR) method which takes into consideration the physical slice acquisition process, ii) use of a novel SRR framework to reconstruct upper abdominal MRI using a single, consistent model to incorporate the PSF in both registration and reconstruction steps and iii) novel use of an existing heavily T2-weighted volume available in MRCP studies to guide registration.

\section{PSF-Aware Slice-to-Volume Registration for SRR}

In this section, we describe the proposed framework for reconstructing the upper abdominal anatomy based on two orthogonal single-shot T2-weighted stacks and a heavily T2-weighted volume ("3D reference") to guide the motion correction. We use a single, uniform approach which incorporates a PSF-aware model for both the registration and reconstruction steps. Additionally, we apply an efficient scattered data approximation approach to initialize the SRR algorithm with a regular grid volume from scattered slices.

Slice Acquisition Model and Algorithm Overview. Starting from the classical slice acquisition model $[5,7]$

$$
\mathbf{y}_{k}=\mathbf{D}_{k} \mathbf{B}_{k} \mathbf{W}_{k} \mathbf{x}+\mathbf{n}_{k}
$$


a relationship between the (vectorized) acquired LR $2 \mathrm{D}$ slice $\mathbf{y}_{k} \in \mathbb{R}^{N_{k}}$ and the unknown (vectorized) HR volume $\mathbf{x} \in \mathbb{R}^{N}$ can be established whereby $N_{k} \ll N$ due to the LR $2 \mathrm{D}$ image acquisition. The remaining variables in (1) include the linear downsampling operator $\mathbf{D}_{k}$, the linear blurring operator $\mathbf{B}_{k}$ carrying the PSF information, the linear motion operator $\mathbf{W}_{k}$ and the image noise $\mathbf{n}_{k} \in \mathbb{R}^{N}$ for each slice $k \in\{1, \ldots, K\}$, respectively.

The following is assumed: i) the resolution of the heavily T2-weighted volume is sufficiently high to act as a 3D reference volume, ii) the occurring deformation can be captured by deforming the slice only in the in-plane direction; the contribution in the orthogonal slice-select direction can therefore be neglected given the thick slices and the associated intensity information uncertainty due to PVEs. Based on those assumptions, we propose the following non-iterative 3step motion correction algorithm for upper abdominal anatomy whereby each step fully respects the assumed physical acquisition model (1):

1. Multimodal volume-to-volume registration: Rigid registration is applied between each stack and the 3D reference.

2. Multimodal slice-to-volume registration: Each individual slice of each stack is rigidly registered to the $3 \mathrm{D}$ reference.

3. In-plane deformation: Based on the intersection of the slices with the 3D reference, each slice is deformed in-plane to compensate for non-rigid deformations.

A volumetric reconstruction based on Tikhonov regularization is then applied. In summary, the algorithm only requires one motion correction cycle consisting of three steps to register the slices with the heavily $\mathrm{T} 2$-weighted volume before one SRR step is performed to reconstruct a single, isotropic HR volume from motion corrected, scattered, single-shot slices.

Point-Spread-Function-Aware Slice-to-Volume Registration. The intent of using a PSF-aware registration is to blur the moving image (3D reference) with the PSF defined by the relative position between fixed image (LR 2D slice) and moving image in order to make them comparable during the registration process $[2,3]$. However, although the classical slice acquisition model provides an intuitive understanding about the physical process, the direct computation with the large matrices involved would cause a substantial memory cost even for sparse representation. Avoiding the explicit storage of matrix-coefficients allows for a more efficient iterative computational scheme [4]. Therefore, we chose to represent (1) pointwise as a matrix-free formulation

$$
\mathbf{y}_{k}(i)=A_{k}(i, \mathbf{x}) \in \mathbb{R} \quad \text { for all } i=1, \ldots, N_{k}
$$

with a linear operator $A_{k}(i, \cdot)$ acting as PSF-defined intensity interpolator in the floating space at a (transformed) physical position of voxel $i$ of slice $\mathbf{y}_{k}$. The PSF itself is defined by the MR acquisition protocol. In practice, a reasonable approximation for single-shot sequences in the slice-coordinate system 
has been found to be a 3D Gaussian defined by the variance-covariance ma$\operatorname{trix} \widetilde{\mathbf{B}}_{k}:=\operatorname{diag}\left(\frac{\left(1.2 s_{1}\right)^{2}}{8 \ln (2)}, \frac{\left(1.2 s_{2}\right)^{2}}{8 \ln (2)}, \frac{s_{3}^{2}}{8 \ln (2)}\right)$ with $s_{1}, s_{2}$ being the spacing in-plane and $s_{3}$ through-plane $[2,6]$. For the registration, this variance-covariance matrix needs to be expressed in the coordinate system of the moving image in order to accommodate the interpolation in the moving space accordingly. Slices are rigidly motion corrected to find the best rigid motion estimate within the $3 \mathrm{D}$ reference before the non-rigid deformation step is applied. Hence, a basis transform with orthogonal matrix $\mathbf{U}_{k}$, accounting for the rotation between the LR slice and the HR volume, expresses the PSF by $\mathbf{U}_{k}^{T} \widetilde{\mathbf{B}}_{k} \mathbf{U}_{k}$ for each single point with respect to the floating space. That means, a PSF-aware SVR can be implemented by providing an oriented Gaussian interpolator $A_{k}$ for each slice $k$ to a generic registration framework which updates the PSF depending on the current transformation parameters. The operation $A_{k}(i, \cdot)$ can be efficiently computed as a matrix-vector multiplication without storing a matrix explicitly by iterating over the $N_{k} \ll N$ voxels in a multi-threaded fashion while considering the oriented Gaussian-weighted 3D reference volume voxel intensities.

Similarly, the multimodal volume-to-volume registration is made PSF-aware by blurring the 3D reference with an oriented Gaussian filter considering the PSF defined by slice-select direction and slice dimensions for each stack.

Super-Resolution Reconstruction. Once the slices are PSF-aware motion corrected, a reconstruction step based on (1) and similar to that described by [5] can be deployed. With a mask operator $\mathbf{M}_{k}$ used to select the region of interest within each slice $k$, the minimization problem reads

$$
\min _{\mathbf{x}}\left(\sum_{k=1}^{K} \frac{1}{2}\left\|\mathbf{M}_{k}\left(\mathbf{y}_{k}-\mathbf{A}_{k} \mathbf{x}\right)\right\|_{\ell^{2}}^{2}+\frac{\alpha}{2}\|\mathbf{D} \mathbf{x}\|_{\ell^{2}}^{2}\right) \quad \text { subject to } \quad \mathbf{x} \geq 0
$$

where $\mathbf{A}_{k} \mathbf{x}$ denotes the application of (2) stacked to a vector in $\mathbb{R}^{N_{k}}, \alpha$ the regularization parameter and $\mathbf{D}$ the differential operator applied on the HR reconstruction estimate $\mathbf{x}$. In this application, we chose a L-BFGS-B algorithm to deal with this large linear system and its positivity constraints to solve the corresponding normal equations. The required adjoint oriented Gaussian operator $\mathbf{A}_{k}^{*}$ can be computed in a similar matrix-free fashion as $\mathbf{A}_{k}$, which can be shown by elementary transformations. Using a first-order Tikhonov regularization term in (3) has the advantage of introducing a correlation between neighbouring voxels, which is especially useful since only two orthogonal stacks with thick slices are available and it is likely that certain areas of the volume are not sufficiently sampled after having registered each slice individually to the $3 \mathrm{D}$ reference.

Scattered Data Approximation. In order to initialize the SRR solver with a regular grid volume from motion corrected slices we propose a scattered data approximation (SDA) approach. We use a discrete variant of Nadaraya-Watson kernel regression as an efficient SDA scheme for irregularly sampled inputs [14]. 
It is based on nearest neighbour sampling onto a regular grid followed by a subsequent Gaussian blurring operation for each single slice.

\section{Data, Evaluation Methodology and Results}

Data and Data Preprocessing. MRCP studies of four anonymized patients, scanned at the University College London Hospital, London, were used for this study. Among the clinically acquired scans for MRCP studies, a set of axial and coronal 2D HASTE sequences and a 3D heavily T2-weighted SPC RST volume acquisition were performed, as shown in fig. 1. The acquisition parameters for the coronal stack were $\mathrm{TE}=91 \mathrm{~ms}, \mathrm{TR}=1350 \mathrm{~ms}$, flip angle of $170^{\circ}$ with resolution of $1.25 \mathrm{~mm} \times 1.25 \mathrm{~mm} \times 6 \mathrm{~mm}$. The respective parameters for the axial stack were $\mathrm{TE}=91 \mathrm{~ms}, \mathrm{TR}=1200 \mathrm{~ms}$, flip angle of $160^{\circ}$ with resolution of $1.48 \mathrm{~mm} \times$ $1.48 \mathrm{~mm} \times 5.50 \mathrm{~mm}$. The heavily T2-weighted volume was acquired in coronal direction with dimensions of $1.09 \mathrm{~mm} \times 1.09 \mathrm{~mm} \times 1.30 \mathrm{~mm}$. HASTE images were preprocessed via an ITK bias field correction filter step [12]. Rectangular masks were provided for both axial and coronal stacks to mark a region of clinical interest.

Parametrization of Reconstruction Pipeline. Both the multimodal volumeto-volume and slice-to-volume PSF-aware registration approaches use mutual information as the similarity measure and are implemented in ITK. The PSF-aware in-plane deformation was performed with the NIFTYREG software using a fast free-form deformation algorithm [9]. Given the different acquisition parameters of the HASTE sequences, a linear model was used for intensity normalization prior to the volumetric reconstruction. The corresponding SRR step was performed with the regularization parameter $\alpha=0.03$ selected via L-curve studies. The initial value was computed by the SDA approach with $\sigma=4$ to avoid the problem of inpainting during SRR.

Evaluation Methodology. The algorithm was run with and without the consideration of the oriented PSF for all registration steps $\left(\mathrm{PSF}_{0}\right.$ or $\left.\mathrm{PSF}_{1}\right)$ and with and without usage of the in-plane deformation model $\left(\mathrm{DM}_{0}\right.$ or $\left.\mathrm{DM}_{1}\right)$ resulting in four different reconstructions for analysis. The reconstructions were initially quantitatively assessed by evaluating the residual via a normalized cross correlation metric, instead of the $\ell^{2}$-norm, in order to be insensitive to the intensity normalization step used to compensate for the different acquisition protocols. Following this, subjective assessment in a clinical context was made including direct comparison to reconstructions obtained by open-source toolkits successfully employed in the challenging problem of fetal MRI reconstructions (BTKtoolkit [11], version from 6 Jan 2016, and the IRTK-based toolkit [7], version from 11 Jun 2015). Two radiologists, blinded to the reconstruction methods, individually assessed reconstruction side-by-side and in comparison to the original HASTE data. The final score is a joint agreement of the radiologists' individual results. Scores were given for: 
1. Clinical usefulness: based on how well common bile duct (CBD), left and right hepatic duct (LHD \& RHD) were visualized and the degree of visible motion artefacts

2. Reconstruction quality: inferred by assessment of preservation of original structural information and the amount of additionally introduced artefactual structures

3. Radiologists' preferred reconstruction

Results. The evaluation of the residuals (1) for all four subjects are visualized in fig. 2. The best agreement between the observed slice $\mathbf{y}_{k}$ and simulated slice $\mathbf{M}_{k} \mathbf{A}_{k} \mathbf{x}$ was obtained for the reconstruction which used the most comprehensive model including PSF-aware registration and in-plane deformable model $\left(\mathrm{PSF}_{1} \mathrm{DM}_{1}\right)$. This is confirmed by calculating the mean of the residuals which rank $\mathrm{PSF}_{1} \mathrm{DM}_{1}$ ahead of all other variants. $\mathrm{PSF}_{1} \mathrm{DM}_{1}$ yields consistently better agreement for subjects 3 and 4 compared to other approaches which show less accurate registration results for some slices.

The radiologists' evaluation, shown in table 1, indicates that the blinded radiologists had a clear preference for our novel PSF-aware SVR reconstructions. Additionally, our proposed reconstruction framework yields reconstructions of similar clarity of CBD, LHD and RHD as the original HASTE data. The reconstructions obtained via IRTK score slightly lower and it was felt that the images would not be suitable for making a clinical diagnosis. Furthermore, all reconstruction approaches demonstrate their ability to correct for motion visible in the HASTE data. With regards to preservation of information in the orginal HASTE stacks, our novel PSF-aware SVR reconstructions are close to the originals' whereas IRTK and BTK $^{5}$ perform less satisfactorily. All reconstruction methods, to some degree, introduce structures which cannot be directly visualized by the original HASTE data.

In fig. 3 our reconstruction variant $\mathrm{PSF}_{1} \mathrm{DM}_{1}$ and the reconstructions based on IRTK and BTK of one subject are provided along with the linearly resampled original data for comparison. This demonstrates that our proposed reconstruction framework largely preserves axial and coronal HASTE data information with minor degradation in image quality as opposed to both IRTK and BTK reconstructions. Moreover, it reveals sharp tissue delineation also in sagittal section where no image stack information is provided.

\section{Discussion}

In this work, we present for the first time a single, consistent SRR framework which takes into consideration the PSF for both the motion correction and vol-

\footnotetext{
${ }^{5}$ The BTK-results used in here do not include the SRR step. Using the standard parametrization of BTK, the SRR outcome was less satisfying and of poorer quality than the reconstruction obtained via local neighbourhood oriented Gaussian interpolation [10].
} 

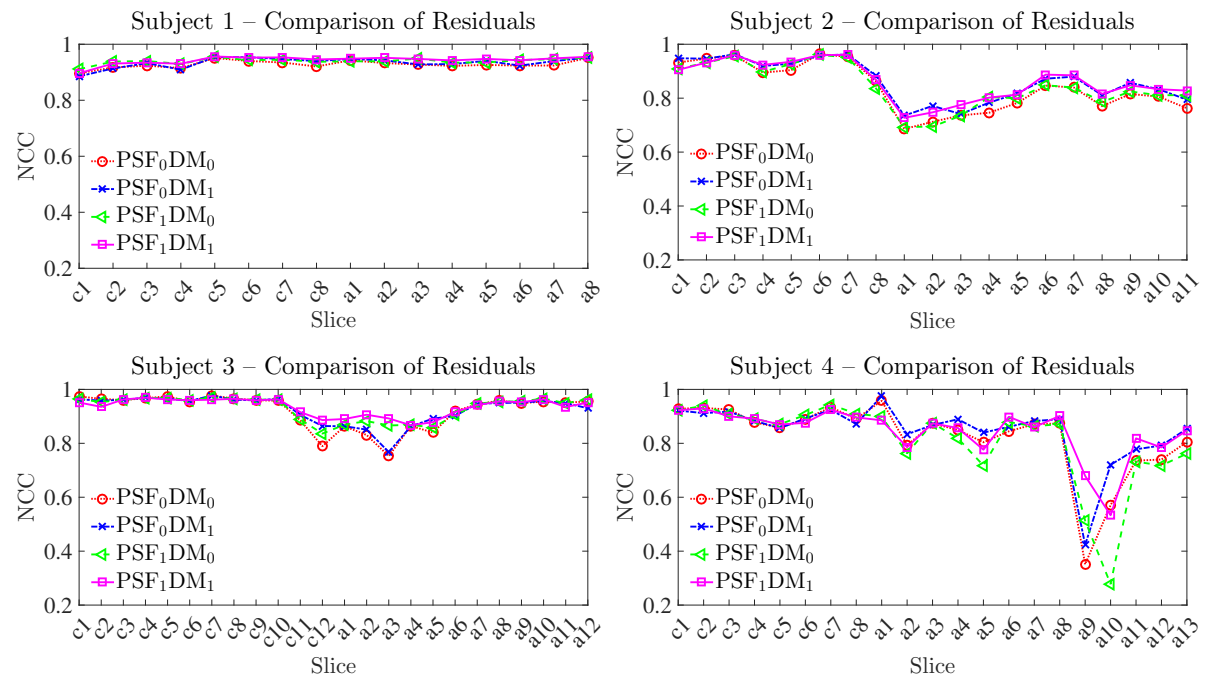

Fig. 2. Evaluation of the residuals for all subjects and modes of our proposed reconstruction framework visualized for all axial slices (a) and coronal slices (c). The associated NCC mean and standard deviation over all subjects for each mode are $0.88 \pm 0.10$ for $\mathrm{PSF}_{0} \mathrm{DM}_{0}, 0.89 \pm 0.08$ for $\mathrm{PSF}_{0} \mathrm{DM}_{1}, 0.88 \pm 0.11$ for $\mathrm{PSF}_{1} \mathrm{DM}_{0}$ and $0.90 \pm 0.08$ for $\mathrm{PSF}_{1} \mathrm{DM}_{1}$, respectively.

Table 1. Summary of clinical evaluation averaged over all four subjects. Evaluation included original HASTE data, four modes of our proposed reconstruction framework and reconstructions by other toolkits (BTK, IRTK). Clarity of anatomical structure score indicates how well CBD, LHD and RHD are visualized in each image with ratings 0 (structure not seen), 1 (poor depiction), 2 (suboptimal visualization; image not adequate for diagnostic purposes), 3 (clear visualization of structure but reduced tissue contrast; image-based diagnosis feasible) and 4 (excellent depiction; optimal for diagnostic purposes). Visible motion score rates the amount of visible non-corrected motion from score 0 (complete motion) to 3 (no motion). Preserved structural information score indicates how well original HASTE data information has been preserved with grades 0 (structures not identified), 1 (poor visualization of structures), 2 (clear visualization but not as good as originals) and 3 (as good as original). Introduced artefacts score rates the amount of additional artefactual structures from 0 (lots of new artefacts) to 2 (no new artefact). Radiologists' preference ranks the subjectively preferred reconstructions from 1 (least preferred) to 6 (most preferred) reconstruction.

\begin{tabular}{lccccc} 
& \multicolumn{2}{c}{ Clinical Usefulness } & \multicolumn{2}{c}{ Reconstruction Quality } & \\
& $\begin{array}{c}\text { Clarity of } \\
\text { Anatomical } \\
\text { Structures }\end{array}$ & $\begin{array}{c}\text { Visible } \\
\text { Motion }\end{array}$ & $\begin{array}{c}\text { Preserved } \\
\text { Structural } \\
\text { Information }\end{array}$ & $\begin{array}{c}\text { Introduced } \\
\text { Artefacts }\end{array}$ & $\begin{array}{c}\text { Radiologists' } \\
\text { Preference }\end{array}$ \\
\hline HASTE & $2.9 \pm 0.3$ & $1.8 \pm 0.5$ & - & - & - \\
Ax \& Cor & $2.9 \pm 0.3$ & $2.8 \pm 0.5$ & $2.0 \pm 0.0$ & $0.8 \pm 0.5$ & $4.2 \pm 0.9$ \\
$\mathbf{P S F}_{\mathbf{0}} \mathbf{D M}_{\mathbf{0}}$ & $2.9 \pm 0.5$ & $1.0 \pm 0.0$ & $5.5 \pm 1.0$ \\
$\mathbf{P S F}_{\mathbf{0}} \mathbf{D M}_{\mathbf{1}}$ & $2.9 \pm 0.3$ & $2.5 \pm 0.5$ & $1.8 \pm 0.5$ & $0.5 \pm 0.5$ & $3.5 \pm 1.0$ \\
$\mathbf{P S F}_{\mathbf{1}} \mathbf{D M}_{\mathbf{0}}$ & $2.9 \pm 0.3$ & $2.8 \pm 0.5$ & $2.0 \pm 0.0$ & $0.5 \pm 0.5$ & $4.5 \pm 0.5$ \\
$\mathbf{P S F}_{\mathbf{1}} \mathbf{D M}_{\mathbf{1}}$ & $2.9 \pm 0.3$ & $2.8 \pm 0.5$ & $2.0 \pm 0.0$ & $0.0 \pm 0.0$ & $1.8 \pm 0.5$ \\
$\mathbf{I R T K}_{\mathbf{B T K}}$ & $2.4 \pm 0.5$ & $2.8 \pm 0.5$ & $1.2 \pm 0.1$ & $1.0 \pm 0.0$ & $1.2 \pm 0.5$
\end{tabular}



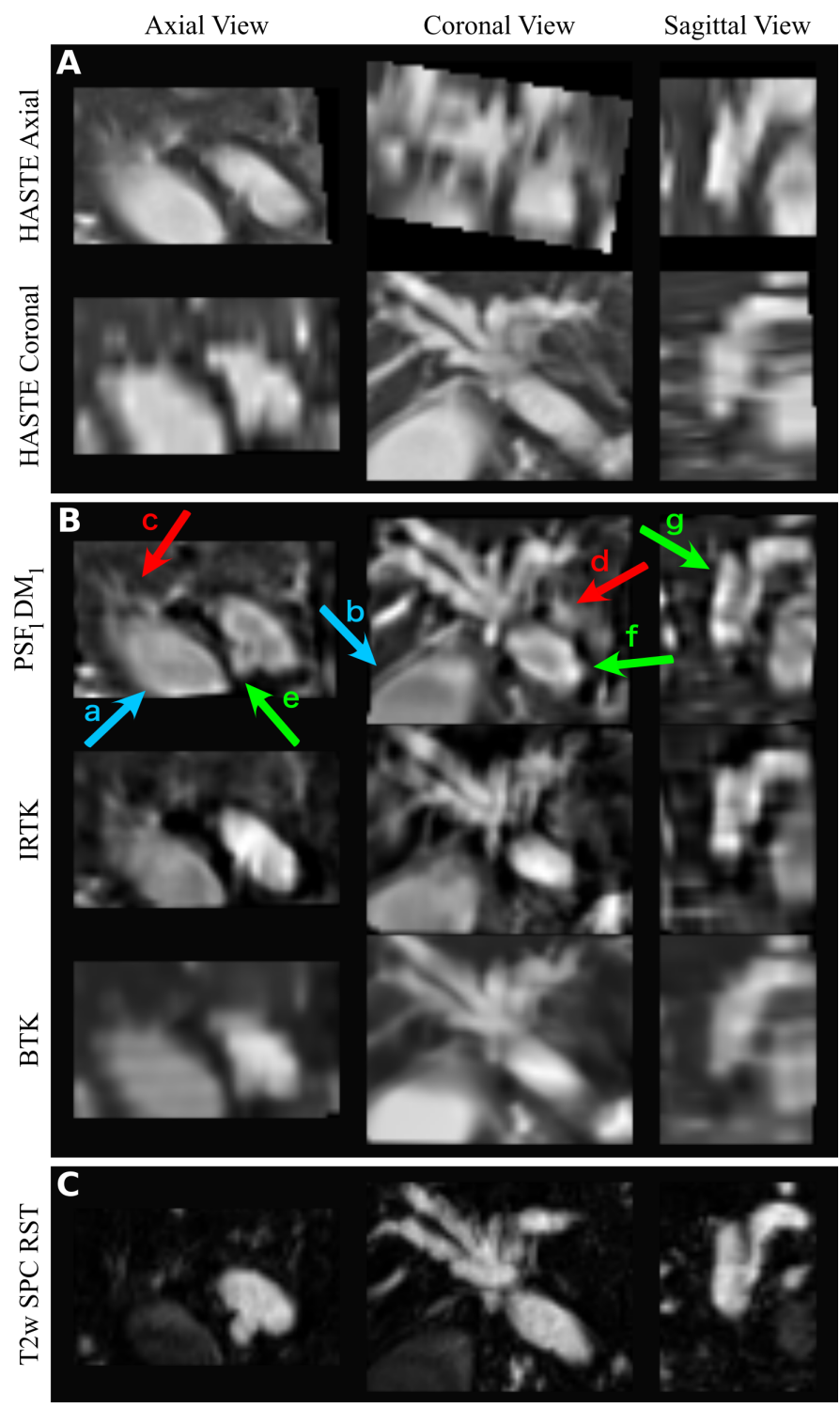

Fig. 3. Qualitative comparison between linearly resampled original HASTE data (A) and reconstructions obtained by BTK, IRTK and our proposed approach (B). Reconstructions are based on one axial and one coronal HASTE stack only. Several arrows on our reconstruction show examples of successfully preserved raw data information (blue $a$ and $b$ ), introduction of artefacts ( $r e d c$ and $d$ ) and resolution improvement (green $e, f$ and $g$ ) in direct comparison with the other reconstruction approaches. Artefacts are explained by similar intensities in the original data $(c)$ in addition to the complex deformation occurred between axial and coronal stack acquisition $(d)$. Resolution improvement was achieved by the combined usage of SR and the incorporated heavily $\mathrm{T} 2$-weighted volume information $(\mathrm{C})$ as reference during motion correction. 
umetric reconstruction steps. We put a particular focus on efficient implementation details like the matrix-free approach to efficiently compute the oriented Gaussian and adjoint oriented Gaussian operators for slice-to-volume registration and the PSF-aware volume-to-volume registration step. We test our framework by reconstructing upper abdominal MRI purely based on existing data available in current clinical MRCP studies. We propose a novel motion correction approach by using the existing heavily T2-weighted volume to guide the slice-to-volume registration to address the challenge of having only two orthogonal stacks with thick slices affected by deformable motion. Despite the high degree of undersampling, we achieve remarkable results which outperform current state-of-the-art techniques developed for fetal MRI, as shown in fig. 3. Further improvements in the current implementation include the incorporation of the oriented PSF for the gradient computation. This shortcoming could also describe the drop in accuracy for some slices observed in fig. 2. Overall, the obtained results are promising and may have the potential to avoid CT scans for further evaluation of this area. Existing limitations are assuming and only accounting for in-plane deformation and sparseness of available data. In the future, we expect to make further improvements using more orthogonal stacks for higher anatomy sampling in combination with a more refined motion model. This will also allow increasing the field of view of the reconstruction to assess the entire biliary tree of clinical interest.

\section{Acknowledgements}

This work is supported by the EPSRC-funded UCL Centre for Doctoral Training in Medical Imaging (EP/L016478/1), the Department of Healths NIHR-funded Biomedical Research Centre at University College London Hospitals and Innovative Engineering for Health award by the Wellcome Trust [WT101957] and Engineering and Physical Sciences Research Council (EPSRC) [NS/A000027/1]. Furthermore, this work was funded by NIHR Clinical Lectureship and NIHR Senior Investigator grant.

\section{References}

1. Barish, M.A., Yucel, E.K., Ferrucci, J.T.: Magnetic Resonance Cholangiopancreatography. New England Journal of Medicine 341(4), 258-264 (1999)

2. Cardoso, M.J., Modat, M., Vercauteren, T., Ourselin, S.: Scale Factor Point Spread Function Matching: Beyond Aliasing in Image Resampling. In: Medical Image Computing and Computer-Assisted Intervention - MICCAI 2015, pp. 675-683. Springer International Publishing (2015)

3. Chacko, N., Chan, K.G., Liebling, M.: Intensity-based point-spread-function-aware registration for multi-view applications in optical microscopy. In: 2015 IEEE 12th International Symposium on Biomedical Imaging (ISBI). pp. 306-309. IEEE (2015)

4. Diamond, S., Boyd, S.: Convex Optimization with Abstract Linear Operators. In: IEEE International Conference on Computer Vision (ICCV). pp. 675-683. No. 1, IEEE (2015) 
5. Gholipour, A., Estroff, J.A., Warfield, S.K.: Robust Super-Resolution Volume Reconstruction From Slice Acquisitions: Application to Fetal Brain MRI. IEEE Transactions on Medical Imaging 29(10), 1739-1758 (2010)

6. Jiang, S., Xue, H., Glover, A., Rutherford, M., Rueckert, D., Hajnal, J.V.: MRI of Moving Subjects Using Multislice Snapshot Images With Volume Reconstruction (SVR): Application to Fetal, Neonatal, and Adult Brain Studies. IEEE Transactions on Medical Imaging 26(7), 967-980 (2007)

7. Kainz, B., Steinberger, M., Wein, W., Kuklisova-Murgasova, M., Malamateniou, C., Keraudren, K., Torsney-Weir, T., Rutherford, M., Aljabar, P., Hajnal, J.V., Rueckert, D.: Fast Volume Reconstruction From Motion Corrupted Stacks of 2D Slices. IEEE Transactions on Medical Imaging 34(9), 1901-1913 (2015)

8. McClelland, J.R., Hawkes, D.J., Schaeffter, T., King, A.P.: Respiratory motion models: A review. Medical Image Analysis 17(1), 19-42 (2013)

9. Modat, M., Ridgway, G.R., Taylor, Z.A., Lehmann, M., Barnes, J., Hawkes, D.J., Fox, N.C., Ourselin, S.: Fast free-form deformation using graphics processing units. Computer Methods and Programs in Biomedicine 98(3), 278-284 (2010)

10. Rousseau, F., Glenn, O.A., Iordanova, B., Rodriguez-Carranza, C., Vigneron, D.B., Barkovich, J.A., Studholme, C.: Registration-Based Approach for Reconstruction of High-Resolution In Utero Fetal MR Brain Images. Academic Radiology 13(9), 1072-1081 (2006)

11. Rousseau, F., Oubel, E., Pontabry, J., Schweitzer, M., Studholme, C., Koob, M., Dietemann, J.L.: BTK: An open-source toolkit for fetal brain MR image processing. Computer Methods and Programs in Biomedicine 109(1), 65-73 (2013)

12. Tustison, N.J., Avants, B.B., Cook, P.A., Zheng, Y., Egan, A., Yushkevich, P.A., Gee, J.C.: N4ITK: Improved N3 Bias Correction. IEEE Transactions on Medical Imaging 29(6), 1310-1320 (2010)

13. Van Reeth, E., Tan, C.H., Tham, I.W., Poh, C.L.: Isotropic Reconstruction of a 4-D MRI Thoracic Sequence Using Super-Resolution. Magnetic Resonance in Medicine 73(2), 784-793 (2015)

14. Vercauteren, T., Perchant, A., Malandain, G., Pennec, X., Ayache, N.: Robust mosaicing with correction of motion distortions and tissue deformations for in vivo fibered microscopy. Medical Image Analysis 10(5), 673-692 (2006)

15. Woo, J., Murano, E.Z., Stone, M., Prince, J.L.: Reconstruction of High-Resolution Tongue Volumes From MRI. IEEE Transactions on Biomedical Engineering 59(12), 3511-3524 (2012) 